\title{
Cross-Layer Radio Resource Allocation in Packet CDMA Wireless Mobile Networks with LMMSE Receivers
}

\author{
Fei Yu and Vikram Krishnamurthy \\ Department of Electrical and Computer Engineering, \\ The University of British Columbia, \\ 2356 Main Mall, Vancouver, BC, Canada V6T $1 Z 4$ \\ ffeiy, vikramk\}aece.ubc.ca
}

\begin{abstract}
Most of previous study of radio resource allocation in traditional wireless networks concentrates on network layer connection blocking probability QoS. In this paper, we show that physical layer techniques and QoS have significant impact on network layer QoS. We define a novel concept of cross-layer effective bandwidth and use this to measure the unified radio resource usage taking into account both physical layer linear minimum-mean square error (LMMSE) receivers and varying statistical characteristics of the packet traffic in code devision multiple access (CDMA) networks. We demonstrate the similarity between traditional circuit-switched networks and packet CDMA networks, which enables rich theories developed in traditional wireless networks to be used in packet CDMA networks. Moreover, since both physical layer signal-to-interference ratio (SIR) QoS and network layer connection blocking probability QoS are considered simultaneously, we can explore the tradeoff between physical layer QoS and network layer QoS in packet CDMA networks.
\end{abstract}

\section{Introduction}

An efficient resource allocation scheme is crucial for guaranteeing different quality of service (QoS) requirements and fully utilizing the scarce radio resource available in wireless mobile networks. Several schemes have recently been proposed for resource allocation in wireless mobile networks. In [1], the complete sharing (CS) and complete partition (CP) schemes are studied. The CS policy allows all connections equal access to the radio resource at all the time, which will result in maximum usage of the available resource. However, at the same time, it does not provide different network layer QoS (e.g., connection blocking probabilities) to different classes of traffic when traffic load is heavy. The CP policy divides up the available resource into separate sub-pools, and each class of traffic can only access its resource pool. This policy allows for more control of the QoS. In the guard channel scheme [2], [3], a portion of resource is reserved for some important classes (e.g., handoff connections) to provide better QoS to these classes. The fractional guard channel scheme [4] is to admit a less important connection (e.g., new connection) with a certain probability when the system (the number of all ongoing connections) is in certain states. The system state can also be defined as the number 
of ongoing new connections. This leads to the new connection bounding scheme [5]. When the resource is not available, some classes of connection requests can be queued instead of being rejected to provide different QoS to different classes [6], [7]. Authors in [8] investigate the comparative performance of different resource allocation schemes.

Although much work has been done in resource allocation of wireless mobile networks, most of previous work concentrates on network layer QoS, blocking probabilities of new and handoff connections, and does not consider physical layer technologies and physical layer QoS. While the decoupling between network layer and physical layer is appropriate for circuit-switched time division multiple access (TDMA) and frequency division multiple access (FDMA) systems, this approach may not be suitable for packet-switched code division multiple access (CDMA) networks. In fact, the interplay between physical layer and network layer plays an important role in CDMA networks with linear minimum-mean square error (LMMSE) multiuser receivers [9]. Unlike the conventional matched filter receivers, the LMMSE receivers take into account the structure of the interference from other users when demodulating a user, and therefore significantly outperform the conventional matched filter receivers [10]. Moreover, unlike traditional circuit-switched networks, future CDMA networks are required to support packet multimedia traffic, which can change the radio resource requirement during a connection's lifetime. Consequently, both cross-layer interplay and packet traffic in CDMA networks complicate the analysis of radio resource allocation schemes as well as hinder the application of rich theories developed in traditional wireless mobile networks [1]-[8] to packet CDMA networks. To the best of our knowledge, analysis of radio resource allocation schemes that considers both CDMA LMMSE physical layer and packet traffic has not been addressed in previous work. For example, the CDMA capacity in [11], [12] is evaluated under the assumption that matched filter receivers are used to demodulate users, and packet traffic is not considered there. In addition, authors in [9], [13] only consider circuit-switched constant bit rate traffic.

In this paper, we study the cross-layer radio resource allocation problem in packet CDMA networks with LMMSE receivers. The novelties of this work are as follows.

1) A novel concept of cross-layer effective bandwidth [14] is used to measure the unified radio resource usage taking into account both LMMSE receivers and varying statistical characteristics of the packet traffic in CDMA networks. Based on the concept of cross-layer effective bandwidth.

2) Both physical layer signal-to-interference ratio (SIR) QoS and network layer connection blocking probability QoS can be considered simultaneously in radio resource allocation schemes. Therefore, we can explore the tradeoff between physical layer QoS and network layer QoS in packet CDMA networks.

3) Using numerical examples, we show that physical layer receivers have significant impact on the network layer QoS. We also show that the network layer QoS can be improved significantly if the physical layer SIR QoS can be violated with a small probability. This study reveals a number of interesting observations and provides insights into the radio resource allocation problem from a cross-layer perspective.

The rest of the paper is organized as follows. Section 2 describes the traffic model and CDMA model. Section 3 presents the concept of cross-layer effective bandwidth. 
Section 4 discusses cross-layer radio resource allocation schemes. Some numerical examples are given in Section 5. Finally, we conclude this study in Section 6.

\section{Model Description}

In this section, we formulate the radio resource allocation problem in CDMA networks with LMMSE receivers, as shown in Fig. 1. Packet traffic arrivals request to access the CDMA network. A radio resource allocator decides whether or not to admit a user and allocates radio resource to the user if he/she is admitted. Both network layer blocking probability QoS and physical layer SIR QoS are considered in the resource allocation. The admitted users transmit packet traffic over multi-path fading channels. A LMMSE multiuser detector is used to demodulate each user. The SIR and SIR outage probability evaluated at the LMMSE receivers are passed back to the radio resource allocator. We detail the traffic model and the asymptotic system capacity for CDMA networks with LMMSE receivers in the following.

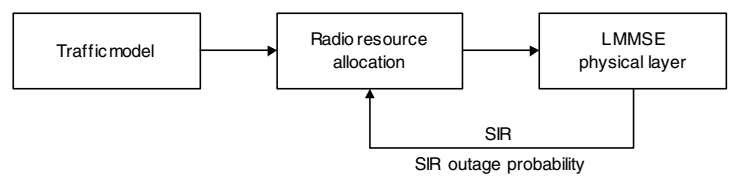

Fig. 1. Cross-layer radio resource allocation in packet CDMA networks with LMMSE receivers

\subsection{Traffic Model}

Assume there are $J$ classes of traffic in the network. The class $j, j=1,2, \ldots, J$, arrival processes of new connections and handoff connections in a cell are Poisson processes with means $\lambda_{j, n}$ and $\lambda_{j, h}$, respectively. We assume that connection holding time for class $j$ connections is exponentially distributed with average value $\mu_{j}$. Each connection transmits packet traffic in the CDMA network. In order to study the characteristics of the packet traffic and propose the cross-layer effective bandwidth concept in Section 3, we introduce the network layer effective bandwidth concept for a given traffic source in wireline networks, which has been well developed [15] during the last decade. Consider a bufferless communication multiplexer with a single output. There are $J$ classes of input traffic with $n_{j}$ connections of class $j$ traffic. The aggregate input traffic is $Y=$ $\sum_{j=1}^{J} \sum_{i=1}^{n_{j}} Y_{j}^{i}$, where $Y_{j}^{i}$ is the $i$ th class $j$ traffic. Assume that the output capacity is $C$. The congestion probability of this system is

$$
P^{\text {cong }}=P\left\{\sum_{j=1}^{J} \sum_{i=1}^{n_{j}} Y_{j}^{i} \geq C\right\} .
$$

Given the statistical characteristics of traffic sources and their congestion probability requirements, the actual bandwidth that a connection requires lies between its mean 
rate and its peak rate. This bandwidth is generally referred to as the effective bandwidth of the traffic source. Assume that $Y_{j}[0, t]$ is the amount of work that arrives from a class $j$ source in the time interval $[0, t]$, and $Y_{j}[0, t]$ has stationary increments. The definition of network layer effective bandwidth of class $j$ traffic is [15]

$$
\alpha_{j}(s, t)=\frac{1}{s t} \log \mathbf{E}\left[e^{s Y_{j}[0, t]}\right], s, t \in \mathbb{R}_{+},
$$

where $\mathbf{E}$ is the expectation, $s$ and $t$ are system parameters defined by the characteristics of the source, its QoS requirements, and the link capacity.

\subsection{Fading Channel and Linear Multiuser Detector Physical Layer Model}

Signal-to-interference ratio (SIR) is the main QoS measure at physical layer. Evaluating the SIR of LMMSE receivers is difficult due to the interwining of the effects of all signature sequences and received powers of all interferers. Fortunately, recent results [10] show that, the SIR can be closely approximated by an expression that only depends on the transmit powers of all active users as well as the first- and second-order statistics of the channel gain, if we assume that signature sequences of the $K$ users are randomly and independently chosen. In this paper, we use these results in radio resource allocation. The path $l$ of user $k$ is characterized by its estimated average channel gain $\bar{h}_{k l}$ and its estimation error variance $\xi_{k}^{2}$. In a large system (both $N$ and $K$ are large), the SIR for the LMMSE receiver of a user (say, the first one) can be expressed approximately as [10] $\operatorname{SIR}_{1}=P_{1} \sum_{l=1}^{L}\left|\bar{h}_{1 l}\right|^{2} \eta /\left(1+P_{1} \xi_{1}^{2} \eta\right)$, where $\eta$ is the unique fixed point in $(0, \infty)$ that satisfies $\eta=\left[\sigma^{2}+1 / N \sum_{k=2}^{K}\left((L-1) I\left(\xi_{k}^{2}, \eta\right)+I\left(\sum_{l=1}^{L}\left|\bar{h}_{k l}\right|^{2}+\xi_{k}^{2}, \eta\right)\right)\right]^{-1}$ and $I(\nu, \eta)=\nu /(1+\nu \eta)$. Assume that there are $J$ classes of traffic in the system. An important physical layer performance measure of class $j$ users is $\operatorname{SIR}_{j}$, which should be kept above the target value $\omega_{j}$. In [13], it is shown that a minimum received power solution exists such that all users in the system meet their target SIRs if and only if $\omega_{j}<\left|\bar{h}_{j}^{i}\right|^{2} / \xi_{j}^{i}$ and

$$
\sum_{j=1}^{J} \sum_{i=1}^{n_{j}} R_{j}^{i} \frac{\Upsilon_{j}^{i}}{N}<1
$$

where $\left|\bar{h}_{j}^{i}\right|^{2}$ is the average channel gain of the $i$ th class $j$ user; $\left|\bar{h}_{j}^{i}\right|^{2}=\sum_{l=1}^{L}\left|\bar{h}_{j l}^{i}\right|^{2}$; $n_{j}$ is the number of class $j$ users; $R_{j}^{i}$ is the number of signature sequences assigned to the $i$ th user of class $j$ to make it transmit at $R_{j}^{i}$ times the basic rate (obtained using the highest spreading gain $N$ ) and

$$
\Upsilon_{j}^{i}=(L-1) \omega_{j} \frac{\xi_{j}^{i^{2}}}{\left|\bar{h}_{j}^{i}\right|^{2}}+\frac{\omega_{j}\left(1+\frac{\xi_{j}^{i 2}}{\left|h_{j}^{i}\right|^{2}}\right)}{1+\omega_{j}} .
$$

Note that multi-code CDMA is used in the above model, in which variable bit rate is provided using multiple codes and the SIR requirement of a connection does not change when the bit rate varies [16]. The capacity of the system is restricted by the 
power control feasibility condition (3). The SIR outage probability in CDMA networks with LMMSE receivers can be expressed as

$$
P^{\text {out }}=P\left\{\sum_{j=1}^{J} \sum_{i=1}^{n_{j}} R_{j}^{i} \frac{\Upsilon_{j}^{i}}{N} \geq 1\right\} .
$$

\section{Cross-Layer Effective Bandwidth}

\subsection{Definition of Cross-Layer Effective Bandwidth}

Comparing (5) with (1), we can see the similarity between CDMA networks with LMMSE receivers and wireline networks. From a mathematical point of view, there is a scalar $\Upsilon_{j}^{i} / N$ besides the packet traffic $R_{j}^{i}$ in (5). It is very interesting to observe that the scalar $\Upsilon_{j}^{i} / N$ contains all the information about the physical layer LMMSE receivers. Since the definition of network layer effective bandwidth (2) is useful in deriving the congestion probability (1), we can develop a concept of cross-layer effective bandwidth to derive the SIR outage probability (5). This motivates us to define the cross-layer effective bandwidth of a traffic source as follows:

Definition 1. Let L denote the number of resolvable paths that each user appears at the receiver, $\left|\bar{h}_{j}^{i}\right|^{2}$ denote the estimated average channel gain and $\xi_{j}^{i}$ denote the channel estimation error variance of the ith class $j$ connections with a SIR target value $\omega_{j}$ in a CDMA system with spreading gain $N$. Let $R_{j}^{i}[0, t]$ denote the amount of work generated from the ith connection of class $j$ in the time interval $[0, t]$, and $R_{j}^{i}[0, t]$ is assumed to have stationary increments. The cross-layer effective bandwidth of this connection is

$$
\alpha_{j}^{i}(L, N, h, \xi, \omega, s, t)=\frac{1}{s t} \log \mathbf{E}\left[e^{s R_{j}^{i}[0, t] \Upsilon_{j}^{i} / N}\right], L, N \in \mathbb{Z}_{+}, h, \xi, \omega, s, t \in \mathbb{R}_{+},
$$

where $\Upsilon_{j}^{i}$ is defined in (4).

\subsection{Properties of Cross-Layer Effective Bandwidth}

We derive some properties of the cross-layer effective bandwidth defined in (6). These properties give some insights into the radio resource allocation problem from a crosslayer perspective.

Proposition 1. If $R_{j}^{1}[0, t], \ldots, R_{j}^{n_{j}}[0, t]$ are $n_{j}$ independent random processes corresponding to the workload from $n_{j}$ class $j$ independent connections and $R_{j}[0, t]$ stands from the workload of the multiplexed system, $R_{j}[0, t]=\sum_{i=1}^{n_{j}} R_{j}^{i}[0, t]$, then we have

$$
\alpha_{j}(L, N, h, \xi, \omega, s, t)=\sum_{i=1}^{n_{j}} \alpha_{j}^{i}(L, N, h, \xi, \omega, s, t) .
$$


Remark: The cross-layer effective bandwidth for the superposition of independent input processes is the sum of the individual cross-layer effective bandwidths. Therefore, the total cross-layer effective bandwidth of all connections in the system is

$$
\alpha(L, N, h, \xi, \omega, s, t)=\frac{1}{s t} \log \mathbf{E}\left[e^{\frac{s}{N} \sum_{j=1}^{J} \sum_{i=1}^{n_{j}} R_{j}^{i}[0, t] \Upsilon_{j}^{i}}\right]=\sum_{j=1}^{J} \sum_{i=1}^{n_{j}} \alpha_{j}^{i}(L, N, h, \xi, \omega, s, t) .
$$

This additive property shows the similarity between traditional circuit-switched networks and packet CDMA networks with LMMSE receivers. Therefore, if we allocate each connection with its cross-layer effective bandwidth, rich theories in [1]-[8] can be used to analyze various radio resource allocation schemes in packet CDMA networks.

Proof: See Appendix.

Proposition 2. $\frac{\Upsilon_{j}^{i}}{N} \frac{\mathbf{E}\left[R_{j}^{i}[0, t]\right]}{t} \leq \alpha_{j}^{i}(L, N, h, \xi, \omega, s, t) \leq \frac{\Upsilon_{j}^{i}}{N} \frac{\bar{R}_{j}^{i}[0, t]}{t}$.

Remark: The effect of network layer varying statistical characteristics of a connection lies between its mean rate and peak rate in the cross-layer effective bandwidth. Instead of allocating packet multimedia connections with their peak rates or mean rates, we can allocate their cross-layer effective bandwidths in CDMA networks, by which the physical layer QoS can be guaranteed and the network utilization can be increased significantly. We will show this with numerical examples.

Proof: See Appendix.

\subsection{Derivation of SIR Outage Probability Using Cross-Layer Effective Bandwidth}

In this subsection, we derive SIR outage probability using cross-layer effective bandwidth, which will be used in Section 4. SIR is an important physical layer QoS measure in CDMA networks. However, guaranteeing the SIR of all connections at all time instants will result in low network utilization, especially when the traffic is bursty. Therefore, we use SIR outage probability as a QoS measure in wireless packet CDMA networks. Instead of guaranteeing the SIR at all time instants, we can guarantee the SIR outage probability. This formulation is motivated by the design of packet-switched wireline networks. It is well known [17] that allocating all connections with their peak rates guarantees no packet loss, but results in the lowest utilization and no multiplexing gain. Therefore, most bandwidth allocation schemes in wireline networks allow a small packet loss probability to increase the network utilization [17]. Similarly, since most applications in wireless networks can tolerate small probability of SIR outage, we use SIR outage probability as a QoS measure in wireless packet CDMA networks and keep it below a target value $\zeta$. The SIR outage probability can be estimated by the following well-known Chernoff bound [18] approximation

$$
P^{\text {out }}=P\left\{\sum_{j=1}^{J} \sum_{i=1}^{n_{j}} R_{j}^{i} \frac{\Upsilon_{j}^{i}}{N} \geq 1\right\} \approx e^{\Lambda(1)}
$$


where $\Lambda(v)=\inf _{s}[s \alpha-s v], \alpha=\sum_{j=1}^{J} \sum_{i=1}^{n_{j}} \alpha_{j}^{i}$, and $\alpha_{j}^{i}$ is the scaled logarithmic moment generating function of the instantaneous work load of the $i$ th class $j$ connection in a packet bufferless CDMA system. $\alpha_{j}^{i}=\lim _{t \rightarrow 0} \alpha_{j}^{i}(L, h, \xi, \omega, s / t, t)=$ $(1 / s) \log \mathbf{E}\left[e^{s R_{j}^{i} Y_{j}^{i} / N}\right]$. The constraint $P^{\text {out }} \leq \zeta$ will be satisfied if the vector $x=$ $\left(n_{1}, n_{2}, \ldots, n_{J}\right)$ lies within the admissible set

$$
X=\left\{x \in \mathbb{Z}_{+}^{J}: \exp \left\{\inf _{s}\left[s\left(\sum_{j=1}^{J} \sum_{i=1}^{n_{j}} \alpha_{j}^{i}-1\right)\right]\right\} \leq \zeta\right\} .
$$

The Chernoff bound (9) can be further refined [19] by adding a prefactor. $P^{\text {out }} \approx$ $1 / s^{*} \sqrt{2 \pi \partial^{2}\left(s^{*} \alpha\right) / \partial s^{2}} e^{\Lambda(1)}$, where $s^{*}$ attains the infimum in (10). The admissible set using the improved bound becomes

$$
X=\left\{x \in \mathbb{Z}_{+}^{J}: \frac{1}{s^{*} \sqrt{2 \pi \frac{\partial^{2}}{\partial s^{2}}\left(s^{*} \sum_{j=1}^{J} \sum_{i=1}^{n_{j}} \alpha_{j}^{i}\right)}} \exp \left[s^{*}\left(\sum_{j=1}^{J} \sum_{i=1}^{n_{j}} \alpha_{j}^{i}-1\right)\right] \leq \zeta\right\} .
$$

\section{Cross-Layer Radio Resource Allocation in Packet CDMA Networks}

Using the concept of cross-layer layer effective bandwidth, we can reduce the complicated packet CDMA networks with LMMSE receivers to traditional circuit-switched networks, and use rich theories developed for traditional networks to analyze various radio resource allocation schemes in packet CDMA networks. In this section, we present the cross-layer global balance equations for general radio resource allocation schemes, from which blocking probabilities and network utilization can be obtained. Then we consider a set of coordinate convex schemes that have a product form of the equilibrium probabilities. We emphasize that all of these schemes are not new. However, none of them considers physical layer QoS in previous study. Our contribution is to apply these schemes in CDMA networks with LMMSE receivers using the concept of cross-layer effective bandwidth. Since physical layer QoS, SIR outage probability (9), is considered in cross-layer effective bandwidth, we can study the radio resource allocation problem with both physical layer and network layer QoS.

\subsection{Cross-Layer Global Balance Equations}

In a packet CDMA network, define the state vector of the system as $x=\left(n_{1}, n_{2}, \ldots, n_{J}\right)$, where $n_{j}, j=1,2, \ldots, J$, denotes the number of class $j$ connections in the system. The state space $X$ in the system is defined in (10) or (11). Note that physical layer SIR outage probability QoS constraint, $P^{\text {out }} \leq \zeta$, is used to restrict the state space of the system. 
Therefore, different physical layer QoS requirements will result in different state spaces, which have significant impact on the network QoS. As we shall see in Section 5, the network QoS can be improved substantially if a small SIR outage probability is introduced compared to the system in which SIR requirements are guaranteed at all time instants. For each given state $x \in X$, an action $a(x)=\left(a_{1}, a_{2}, \ldots, a_{J}\right) \in\{0,1\}^{J}$ is chosen. If $a_{j}(x)=1$, admit a class $j$ connection when the system state is $x$; if $a_{j}(x)=0$, the connection is rejected. The action space is a set of all possible actions, which can be defined as $A=\left\{a: a \in\{0,1\}^{J}, j=1,2, \ldots, J\right\}$. The action is done according to a radio resource allocation scheme $u \in \mathcal{U}$, where $\mathcal{U}$ is defined as $\mathcal{U}=\{u: X \rightarrow A\}$. $\{x(t), u\}_{t \in \mathbb{R}_{+}}$is a Markov process under each radio resource allocation scheme. Let $\pi_{u}(x)$ denote the equilibrium probability that the system is in state $x$ under scheme $u$. Define $e_{j} \in\{0,1\}^{J}$ as a row vector containing only zeros except for the $j$ th component, which is $1 . x+(-) e_{j}$ corresponds to an increase (decrease) of the number of class $j$ connections by 1 . The global balance equations for the Markov Chain under scheme $u$ are [20]

$$
\sum_{j=1}^{J} \pi_{u}\left(x-e_{j}\right) \lambda_{j} a_{j}\left(x-e_{j}\right)+\sum_{j=1}^{J} \pi_{u}\left(x+e_{j}\right) \mu_{j}\left(n_{j}+1\right)=\sum_{j=1}^{J}\left[\lambda_{j} a_{j}(x)+\mu_{j}\left(n_{j}+1\right)\right] \pi_{u}(x),
$$

where $x \in X, \lambda_{j}$ and $\mu_{j}$ are class $j$ connection arrival and departure rates, respectively. These global balance equations can be solved using any linear equation procedure, such as Jacobi and Gauss-Seidel methods. Once the equations are solved, network layer blocking probability QoS, can be directly calculated. The blocking probability for a class $j$ connection is

$$
P_{j}^{b}=\sum_{n \in X_{j}} \pi(n)
$$

where $X_{j} \subseteq X$ is the set of states that system will move out of $X$ with addition of one connection of class $j$. This approach is general enough to be applicable to a variety of radio resource allocation schemes.

As the cardinality of $X$ becomes large, the computation complexity of solving the global balance equations is extensive. It is very difficult, if not impossible, to get feasible solutions in real networks due to the problem of large dimensionality. In the following, we consider a set of coordinate convex schemes that have a product form of the equilibrium probabilities.

\subsection{Coordinate Convex Schemes}

The coordinate convex schemes form several important resource allocation schemes, such as complete sharing, complete partitioning and threshold schemes. It is shown in Chapter 4 of [20] that their equilibrium probabilities have a product form. The name coordinate convex scheme comes from the concept of coordinate convex set. A coordinate convex scheme is characterized by a coordinate convex set, which is any nonempty set $\Delta \subseteq X$ with the following property: if $x \in \Delta$ and $n_{j}>0$ then $x-e_{j} \in \Delta$. In a coordinate convex scheme associated with coordinate convex $\Delta$, a connection arrival 
is admitted to the system if and only if the system state remains in $\Delta$ after the admission. The equilibrium probabilities of the system can be obtained from the the theory of multiservice loss networks.

$$
\pi(n)= \begin{cases}\pi_{0} \prod_{j=1}^{J} \frac{\left(\lambda_{j} / \mu_{j}\right)^{n_{j}}}{n_{j} !}, & \text { if } n \in \Delta \\ 0, & \text { otherwise }\end{cases}
$$

where $\pi_{0}$ is a normalization constant,

$$
\pi_{0}=\frac{1}{\sum_{n \in \Delta} \prod_{j=1}^{J} \frac{\left(\lambda_{j} / \mu_{j}\right)^{n_{j}}}{n_{j} !}} .
$$

\section{$5 \quad$ Numerical Results and Discussions}

In this section, we illustrate the performance of the proposed approaches by numerical examples. The numerical values used for the system parameters in the numerical examples are given in Table 1. A CDMA system with system bandwidth $5 \mathrm{MHz}$ and spreading gain $N=512$ is considered. There are two classes of traffic, voice and MPEG video. $20 \%$ of the traffic arrivals are video connections. The service rates are $\mu_{1}$ and $\mu_{2}$. The voice traffic is modeled as an ON/OFF process. The packet transmission rate in state $\mathrm{ON}$ is $15 \mathrm{kbps}$ corresponding to an equivalent spreading gain 256 . The rate from ON to OFF is the same as the rate from OFF to ON, which is 0.4 , for the voice traffic. A Markov model with two states, NORMAL and BURST, is used for the MPEG traffic [21]. The rate from state NORMAL to state BURST is 0.024 and the rate from state BURST to state NORMAL is 0.076 . The data transmission rate in state NORMAL is $30 \mathrm{kbps}$ corresponding to an equivalent spreading gain 128 and the data transmission rate in state BURST is two times of that in state NORMAL.

We compare the admissible region of a packet CDMA network using LMMSE receivers with that of the traditional scenario in which all users are demodulated by matched filters. Two values of the number of resolvable path are considered, $L=1$ and $L=5$. SIR outage is not allowed in this example. Fig. 2 compares the admissible regions when using matched filters vs. using LMMSE receivers. We notice a significant gain in the admissible region when LMMSE receivers are used, such illustrating that physical layer receivers have a significant impact on network layer QoS. We further show this by presenting the connection blocking probabilities of voice in Fig. 3. It is observed that the connection blocking probabilities of voice can be decreased substantially in the LMMSE cases, which illustrates the importance of considering physical layer techniques in the radio resource allocation problem in packet CDMA networks. We also observe that the simulation results roughly agree with those from the analysis.

Using the concept of cross-layer effective bandwidth, we can study the effects of physical layer SIR outage probability QoS on network layer connection blocking probability QoS. We compare three radio resource allocation schemes, peak rate, mean rate and cross-layer effective bandwidth with a small SIR outage probability. In the peak rate allocation scheme, all connections are allocated with their peak rates to guarantee 
Table 1. Parameters used in numerical examples

\begin{tabular}{c|c|c}
\hline Parameter & Notation & Value \\
\hline \hline target SIR for voice traffic & $\omega_{1}$ & $7 \mathrm{~dB}$ \\
\hline estimated average channel gain for voice traffic & $\left|h_{1}\right|^{2}$ & 1 \\
\hline channel estimation error variance for voice traffic & $\xi_{1}^{2}$ & 0.02 \\
\hline data transmission rate in state ON for voice traffic & $R_{1}$ & $15 \mathrm{kbps}$ \\
\hline service rate for voice traffic & $\mu_{1}$ & 0.005 \\
\hline target SIR for video traffic & $\omega_{2}$ & $10 \mathrm{~dB}$ \\
\hline estimated average channel gain for video traffic & $\left|h_{2}\right|^{2}$ & 1 \\
\hline channel estimation error variance for video traffic & $\xi_{2}^{2}$ & 0.05 \\
\hline data transmission rate in state NORMAL for video traffic & $R_{21}$ & $30 \mathrm{kbps}$ \\
\hline data transmission rate in state BURST for video traffic & $R_{22}$ & $60 \mathrm{kbps}$ \\
\hline service rate for video traffic & $\mu_{2}$ & 0.004 \\
\hline
\end{tabular}

the SIRs of all connections at all time instants, and the SIR outage probability is zero. [9] and [13] are examples of the peak rate scheme. In the mean rate allocation scheme, each connection is allocated with its mean rate. Figs. 4, 5] show the admissible regions and the video connection blocking probabilities, respectively. We can see that physical layer SIR outage probability QoS has significant effects on network layer blocking probability QoS. Although peak rate allocation approach can guarantee physical layer SIR requirements at all the time, it results in the smallest admissible region and the highest blocking probabilities. An interesting observation is that, with a small SIR outage probability 0.005 , the cross-layer effective bandwidth approach can increase the admissible region and decrease the blocking probabilities substantially. The mean rate allocation scheme can further increase the admissible region. However, physical layer SIR outage probability cannot be guaranteed in this scheme, which is more than $50 \%$.

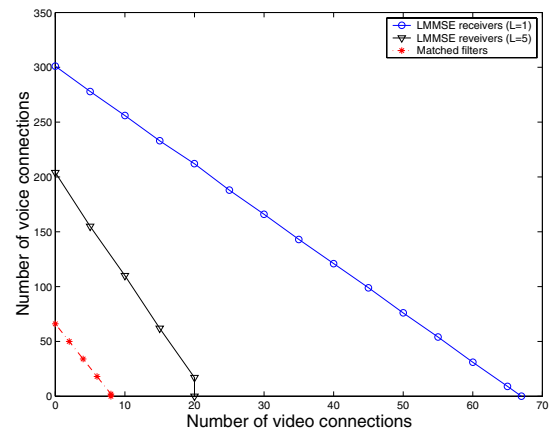

Fig. 2. Admissible regions of different physical layer receivers (SIR outage probability $=0$ )

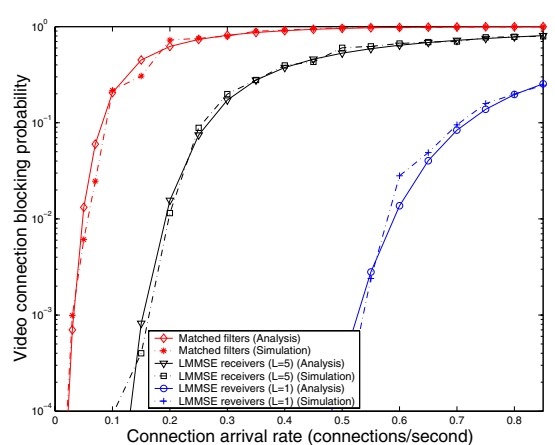

Fig. 3. Voice connection blocking probabilities of different physical layer receivers $($ SIR outage probability $=0)$ 


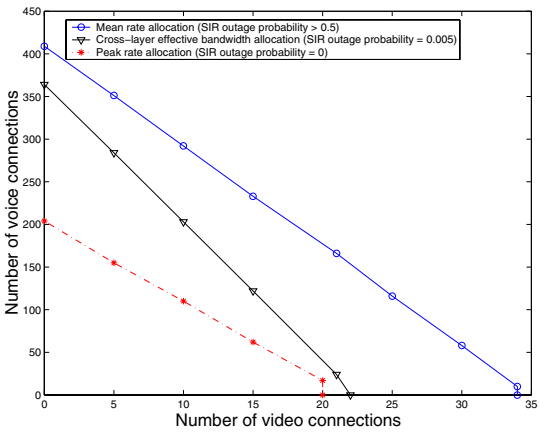

Fig. 4. Admissible regions of different physical layer QoS

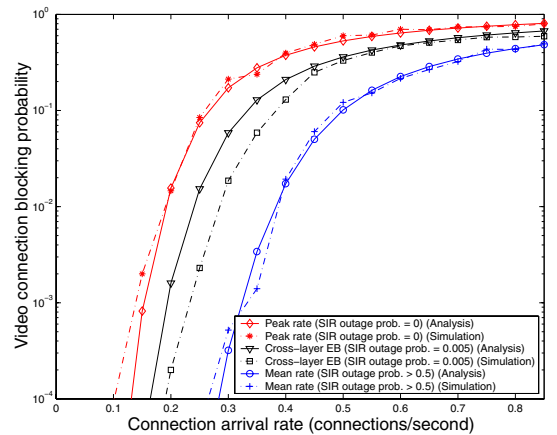

Fig. 5. Video connection blocking probabilities of different physical layer QoS

\section{Conclusions}

We have studied the radio resource allocation problem in packet CDMA wireless mobile networks from a cross-layer perspective. A novel concept of cross-layer effective bandwidth was used for variable bit rate multimedia traffic in packet CDMA networks. Using this concept, we can have a unified measure of resource usage taking into account both physical layer linear minimum mean square error (LMMSE) multiuser receiver structures and varying statistical characteristics of packet traffic. We have shown that physical layer techniques and QoS have significant effects on network layer blocking probability QoS. Substantial performance gain can be achieved using linear minimummean square error (LMMSE) receivers over the scenario in which matched filters are used. It was also observed that network layer QoS can be improved significantly if physical layer QoS can be violated with a small probability.

\section{References}

1. B. Epstein and M. Schwartz, "Reservation strategies for multi-media traffic in a wireless environment," in Proc. IEEE VTC'95, vol. 1, (Rosemont, Illinois), pp. 165-169, July 1995.

2. D. Hong and S. Rappaport, "Traffic model and performance analysis for cellular mobile radio telephone systems with prioritized and nonprioritized handoff procedures," IEEE Trans. Veh. Technol., vol. VT-35, pp. 77-92, Aug. 1986.

3. S. Wu, K. Y. M. Wong, and B. Li, "A dynamic call admission policy with precision QoS guarantee using stochastic control for mobile wireless networks," IEEE/ACM Trans. Networking, vol. 10, pp. 257-271, Apr. 2002.

4. R. Ramjee, D. Towsley, and R. Nagarajan, "On optimal call admission control in cellular network," Wireless Networks, vol. 3, pp. 29-41, Mar. 1997.

5. Y. Fang and Y. Zhang, "Call admission control schemes and performance analysis in wireless mobile networks," IEEE Trans. Veh. Technol., vol. 51, pp. 371-382, Mar. 2002.

6. Y. Lin, S. Mohan, and A. Noerpel, "Queueing priority channel assignment strategies for handoff and initial access for a pcs network," IEEE Trans. Veh. Technol., vol. 43, pp. 704 712, Aug. 1994. 
7. V. Lau and S. Maric, "Mobility of queued call requests of a new call-queueing technique for cellular systems," IEEE Trans. Veh. Technol., vol. 47, pp. 480-488, May 1998.

8. B. Li, L. Li, B. Li, and X. Cao, "On handoff performance for an integrated voice/data cellular system," ACM/Baltzer Wireless Networks, vol. 9, pp. 393-402, July 2003.

9. S. Singh, V. Krishnamurthy, and H. V. Poor, "Integrated voice/data call admission control for wireless DS-CDMA systems with fading," IEEE Trans. Signal Proc., vol. 50, pp. 1483-1495, June 2002.

10. J. Evans and D. N. C. Tse, "Large system performance of linear multiuser receivers in multipath fading channels," IEEE Trans. Inform. Theory, vol. 46, pp. 2059-2078, Sept. 2000.

11. J. Evans and D. Everit, "On the teletraffic capacity of CDMA cellular networks," IEEE Trans. Veh. Technol., vol. 48, pp. 153-165, Jan. 1999.

12. C. Lindemann, M. Lohmann, and A. Thummler, "Adaptive call admission control for QoS/revenue optimization in CDMA cellular networks," ACM/Baltzer Wireless Networks, vol. 10, pp. 457-472, July 2004.

13. C. Comaniciu and H. V. Poor, "Jointly optimal power and admission control for delay sensitive traffic in CDMA networks with LMMSE receivers," IEEE Trans. Signal Proc., vol. 51, pp. 2031-2042, Aug. 2003.

14. F. Yu and V. Krishnamurthy, "Effective bandwidth of multimedia traffic in packet wireless CDMA networks with LMMSE receivers - a cross-layer perspective," IEEE Trans. Wireless Commun., to appear, 2005.

15. F. Kelly, "Notes on effective bandwidths," in Stochastic Networks: Theory and Applications (F. Kelly, S. Zachary, and I. Ziedins, eds.), pp. 141-168, Oxford Press, 1996.

16. C.-L. I and R. D. Gitlin, "Multi-code CDMA wireless personal communications networks," in Proc. IEEE ICC'95, (Seattle, WA), June 1995.

17. M. Schwartz, Broadband Integrated Networks. Prentice Hall, 1996.

18. H. Chernoff, "A measure of asymptotic efficiency for tests of a hypothesis based on the sum of observation," Ann. Math. Statist., vol. 23, pp. 493-507, 1952.

19. R. Bahadur and R. Rao, "On deviations of the sample mean," Ann. Math. Statist., vol. 31, pp. 1051-1027, 1960.

20. K. Ross, Multiservice Loss Models for Broadband Telecommunication Networks. SpringerVerlag, 1995.

21. J. Pechiar, G. Perera, and M. Simon, "Effective bandwidth estimation and testing for markov sources," Performance Evaluation, vol. 48, pp. 157-175, 2002.

\section{Appendix}

Proof (Proposition 3.1). :

$$
\begin{aligned}
& \alpha_{j}=\frac{1}{s t} \log \mathbf{E}\left[e^{s \sum_{i=1}^{n_{j}} R_{j}^{i}[0, t] \Upsilon_{j}^{i} / N}\right]=\frac{1}{s t} \log \mathbf{E}\left[\prod_{i=1}^{n_{j}} e^{s R_{j}^{i}[0, t] \Upsilon_{j}^{i} / N}\right] \\
= & \frac{1}{s t} \log \left(\prod_{i=1}^{n_{j}} \mathbf{E}\left[e^{s R_{j}^{i}[0, t] \Upsilon_{j}^{i} / N}\right]\right)=\sum_{i=1}^{n_{j}} \frac{1}{s t} \log \mathbf{E}\left[e^{s R_{j}^{i}[0, t] \Upsilon_{j}^{i} / N}\right]=\sum_{i=1}^{n_{j}} \alpha_{j}^{i} .
\end{aligned}
$$

Proof (Proposition 3.2). : Using Jensen's inequality,

$$
\begin{aligned}
& \alpha_{j}^{i}=\frac{1}{s t} \log \mathbf{E}\left[e^{s R_{j}^{i}[0, t] \Upsilon_{j}^{i} / N}\right] \geq \frac{1}{s t} \mathbf{E} \log \left[e^{s R_{j}^{i}[0, t] \Upsilon_{j}^{i} / N}\right]=\frac{\Upsilon_{j}^{i}}{N} \frac{\mathbf{E}\left[R_{j}^{i}[0, t]\right]}{t} . \\
& \alpha_{j}^{i}=\frac{1}{s t} \log \mathbf{E}\left[e^{s R_{j}^{i}[0, t] \Upsilon_{j}^{i} / N}\right] \leq \frac{1}{s t} \log \left[e^{s \bar{R}_{j}^{i}[0, t] \Upsilon_{j}^{i} / N}\right]=\frac{\Upsilon_{j}^{i}}{N} \frac{\bar{R}_{j}^{i}[0, t]}{t} .
\end{aligned}
$$

